\title{
Reduced hardness at the onset of plasticity in nanoindented titanium dioxide
}

\author{
V. Navarro, ${ }^{*}$ O. Rodríguez de la Fuente, A. Mascaraque, and J. M. Rojo \\ Departamento de Física de Materiales, Universidad Complutense de Madrid, 28040 Madrid, Spain \\ (Received 14 August 2008; revised manuscript received 14 October 2008; published 19 December 2008)
}

\begin{abstract}
Titanium dioxide rutile crystals have been nanoindented and studied by a combination of atomic force microscopy imaging and analysis of force vs penetration curves. In all the experiments, gold crystals have been used as a reference. A concept of nanoindentation effective volume is introduced to differentiate the bulk behavior from that of a small defect-free volume around the indentation. In the latter volume, a reversible Hertzian elastic stage is identified with a Young modulus comparable to that of the bulk. At higher loads, an incipient plastic range is recognized in which the load is linear on the penetration and permanent traces are left behind at the surface upon tip retraction. In that range, the hardness is constant, about five times smaller than the yield strength and more than three times smaller than the corresponding bulk value. This reduced hardness is explained in terms of the operation of dislocation sources with a low-energy barrier.
\end{abstract}

DOI: 10.1103/PhysRevB.78.224107

PACS number(s): 62.25.-g, 62.20.de, 62.20.fq, 68.47.Gh

\section{INTRODUCTION}

Indentation, involving penetrations in the range of tens of micrometers (in the following designed by microindentation) is the technique of choice to characterize hardness, a key quantity in materials engineering, though not easy to define in unambiguous physical terms. In recent years, nanoindentation, ${ }^{1}$ in which penetrations stay in the range of tens of nanometers and small perturbed volumes are involved around the contact point, has been developed as a technique which probes the surface region. Nanoindentation can indeed give direct empiric information on the mechanical properties of technologically important nanostructures. This is particularly timely in view of the current development of microelectromechanical system (MEMS) devices. However, the information obtained with nanoindentation is, on no account, restricted to the surface region but can be extended to shed light on fundamental mechanisms underlying the physical properties of solids. It is by no means obvious that results obtained with maximum loads extending up to the micrometer range can be straightforwardly extrapolated into the lower end of the nanoscopic range where tip radii and penetrations are on the order of tens of nanometers. For example, mechanical parameters resulting from microindentation-which probes a large volume around the contact - can be taken as representative of the bulk of the real crystal, whereas results from nanoindentation experiments are better explained in terms of an ideal defect-free solid. ${ }^{2}$ The transition from the microindentation to the nanoindentation regime has not been explored in depth, ${ }^{3}$ hampering the interpretation of a number of current experiments.

Most of our present knowledge on surface mechanical properties stems from work in metals: very little research has been carried out in oxides. ${ }^{4}$ Although mechanical properties of metals and oxides differ markedly in the bulk, they have an intriguingly similar response to nanoindentations. When scaling down into the nanoindentation regime, oxides, which in the bulk are known to be brittle, under certain conditions show a ductile response, ${ }^{5}$ in which the nucleation of cracks is averted. Some of these surfaces can even transit between ductile and brittle regimes. ${ }^{6,7}$ Although general trends have been established, much of the underlying physics is still obscure. For example, it is not yet clear whether, in oxides, reversible elastic penetration curves following a Hertzian law, which have been described in many studies in metals, can be identified as such. In addition, the interpretation of the early plastic range-in both type of materials-is open to discussion and, very recently, not less than five different models have been critically assessed vs experimental data ${ }^{8}$ in oxides.

The purpose of the present work is to gain insight into the mechanisms involved in the initial stages of plasticity in oxides and investigate whether the same mechanisms operate in metals. More specifically, the work aims at bridging the gap between the two regimes which limit surface indentation: the one corresponding to nanoindentations which involve relatively small volumes near the surface and the other corresponding to microindentations, and beyond, where the bulk of the solid is probed. There are practically no reports of small-scale nanoindentations performed on oxides focused in the range of penetrations of a few tenths of nanometers carried out with tip radii of less than $50 \mathrm{~nm}$. To this end, we have made a detailed study of nanoindentations, when the volume perturbed thereby is small enough to be clear of preexisting defects. We report on an analysis of $\mathrm{TiO}_{2}$ rutile single crystals nanoindented on their (110) surfaces with tips of small radii, in the range of 30-50 nm, and penetrations of up to a few tens of nanometers. Various stages of the deformation process are identified by a concurrent analysis of force vs penetration curves and atomic force microscopy (AFM) images following tip retraction after nanoindentation. These results are, then, compared with experiments carried out under similar conditions on $\mathrm{Au}(001)$ single crystals. For these small nanoindented volumes, the responses of metal and oxide crystals are found to be remarkably similar. In both types of materials we fully characterize an initial elastic region and, beyond the yield point, reveal the existence of a wide region of penetration in which hardness is constant. When these values of hardness are compared with the corresponding ones on the bulk, qualitative differences between Au and $\mathrm{TiO}_{2}$ show up. A unifying explanation is proposed by 

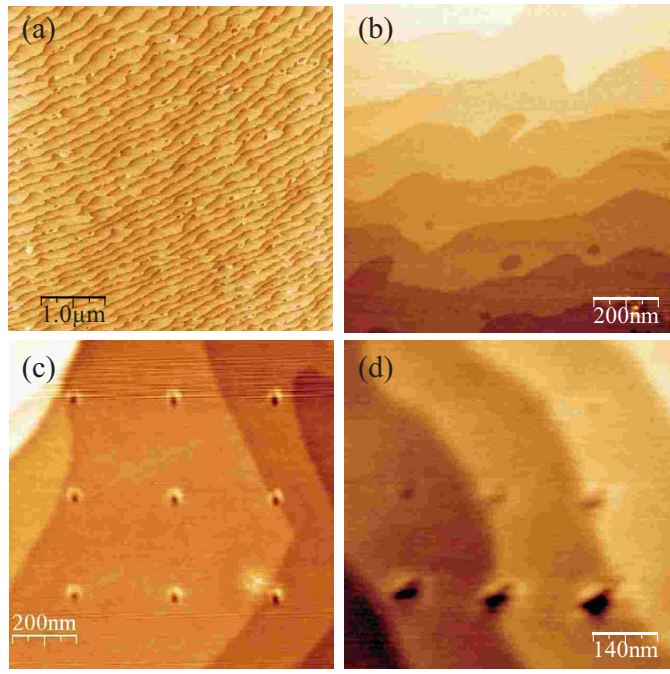

FIG. 1. (Color online) AFM images of $\mathrm{TiO}_{2}(110)$ surface. Panels (a) and (b) correspond to clean sample, where monoatomic steps are clearly observed. In (c) the surface has been indented with a matrix of nine indents, all with the same maximum loads; in (d) the maximum load is successively increased. The first two indents in the first row correspond to the elastic stage (no visible traces) and from the third onward to the transition and plastic regimes. Images in (c) and (d) were taken with the same diamond tip used for indenting, whereas images in (a) and (b) were taken with a sharper silicon tip.

making use of the concept of an effective volume around the nanoindentation.

\section{EXPERIMENT}

$\mathrm{TiO}_{2}(110)$ surfaces were prepared from oriented rutile single crystals by cleaning the sample with a $20 \% \mathrm{HF}$ chemical etching for $10 \mathrm{~min}$ and air annealing for $1 \mathrm{~h}$ at $650{ }^{\circ} \mathrm{C} .{ }^{9}$ In this way, we obtained surface atomically flat terraces between 200 and $300 \mathrm{~nm}$ wide, as can be seen in the AFM images of Fig. 1. In this way, the radius of the nanoindentation traces is small compared to the distance between steps, and, around the center of terraces, the surface can be assumed to be flat. The high quality of the surface is supported by low-energy electron diffraction (LEED) measurements, which give sharp intense $(1 \times 1)$ spots, distinctive of atomically smooth terraces. At the atomic level, steps on the flat terraces show a height of $0.36 \mathrm{~nm}$ [see Fig. 1(b)], in good agreement with the (110) interplanar distances for the unit cell of $\mathrm{TiO}_{2}$. Also, contact AFM images (not included in the paper) show the bridging oxygen rows characteristic of the (110) surface structure with typical periodicity of $0.65 \mathrm{~nm}$. The gold samples were single crystals (Surface Preparation Laboratory), with their normal in the (001) direction. Prior to being transferred into the measurement equipment, they were cleaned under UHV conditions by cycles of successive ion bombardment and annealing at high temperature. All the measurements were performed under ambient conditions. The (001) sample showed large atomically flat terraces (often more than $500 \mathrm{~nm}$ wide) with monoatomic steps. AFM images were treated using the WSXM software from Nanotec. ${ }^{10}$

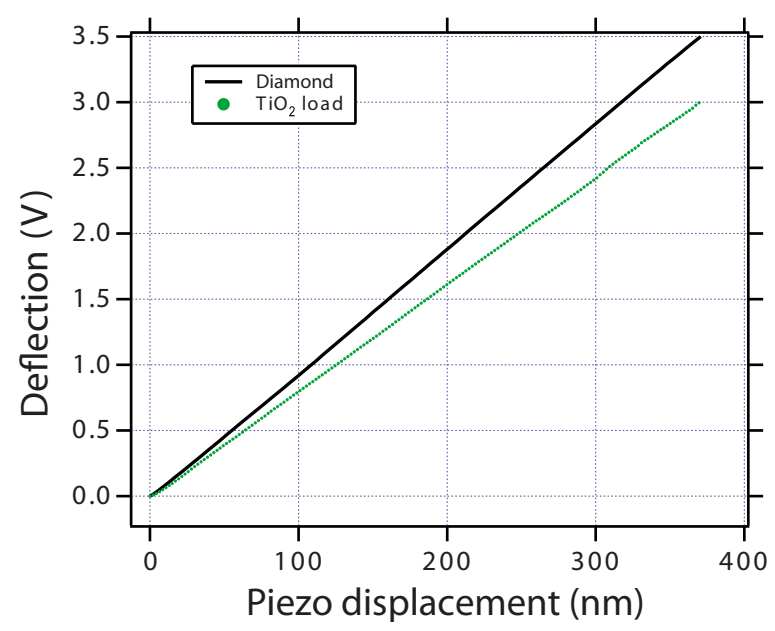

FIG. 2. (Color online) Original cantilever deflection $V$ vs piezodisplacement $\Delta$ for both the $\mathrm{TiO}_{2}$ sample and the diamond reference surface.

Nanoindentation experiments were carried out on $\mathrm{TiO}_{2}$ with an AFM equipped with a Veeco diamond pyramidal tip (nominal radius $R=45 \mathrm{~nm}$ ). Most of the information was derived from force $F$ vs penetration $h$ curves. To obtain these curves, the voltage of the detector (measuring the tip deflection $V$ ) was measured as a function of the displacement of the piezoelectric holding the sample, $\Delta$. To account for the cantilever deflection a reference diamond surface was used. When pushing against this reference surface, the cantilever deflection $V$ was assumed to be due only to piezoelectric displacement $\Delta^{\prime}$. For every value of the deflection voltage, the force $F$ was, then, obtained as $F=k \Delta^{\prime}$ and the effective penetration as $h=\Delta-\Delta^{\prime}$. In this type of study, reproducibility is a key factor: an appreciation of this reproducibility can be obtained by comparing a matrix of nine nanoindentations carried out under the same conditions [Fig. 1(c)]. The size of the traces remaining after tip retraction is practically the same, as it is the extent of material dislodged around the tip.

We have used a Digital Nanoscope III AFM without any tilt compensation. There are some effects reported concerning tilt between tip and sample in nanoindentation experiments. ${ }^{11,12}$ However, from the regular shape of the traces on the AFM images shown in Fig. 1, any tilting effects can be safely ruled out. Also, some precautions were implemented in our data analysis routine: (i) only $F$ vs $z$ curves corresponding to regular traces were used; (ii) large homogeneous regions of the sample were selected for performing the nanoindentations, and finally; (iii) original deflection $V$ vs piezodisplacement $\Delta$ data were carefully inspected to eliminate the curves showing artifacts such as equidistant pop-ins, oscillations, or nonmonotonic behavior. ${ }^{11,12}$ To illustrate the later, a typical original $V$ vs $\Delta$ curve is displayed as Fig. 2. To guarantee that creep effects were not disturbing the results, we performed nanoindentations at different penetration speeds and checked that, within our range of penetrations, there were no recognizable changes in the curves. 


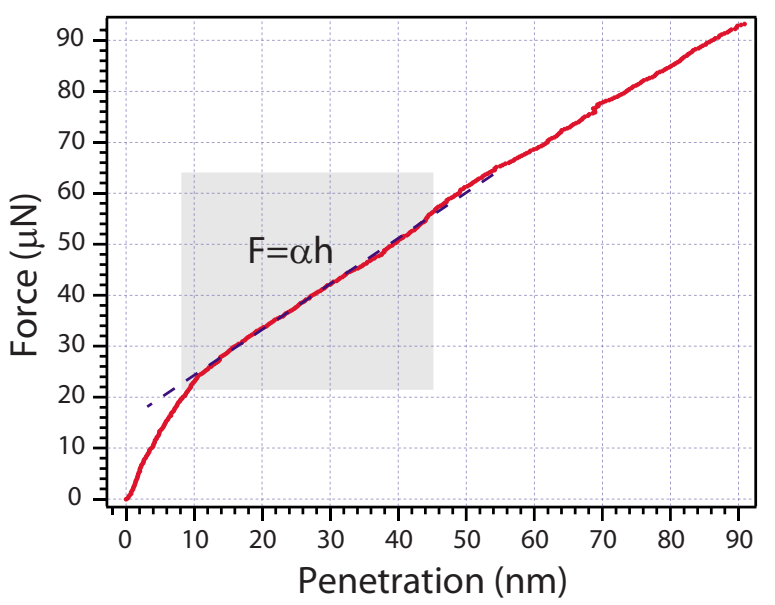

FIG. 3. (Color online) A typical force $F$ vs penetration $h$ curve. The shadowed region corresponds to the incipient plasticity described in the text defined by a hardness $H_{o}$.

\section{RESULTS}

\section{A. Titanium dioxide}

\section{Elastic stage}

A typical loading curve, in which force $F$ (or external load) is represented as a function of penetration $h$, is shown as Fig. 3. It shows two distinct regions. The first one corresponds to penetrations up to around $h=2 \mathrm{~nm}$. A zoom of this region is shown as Fig. 4. As shown by the superimposed line, the points can be fitted to the well-known Hertz formula, ${ }^{13}$

$$
F=\frac{4}{3} R^{1 / 2} E^{*} h^{3 / 2}
$$

$E^{*}$ is the reduced Young modulus for the diamond tip-rutile sample couple, defined by the expression

$$
\frac{1}{E^{*}}=\frac{1-\nu^{2}}{E}+\frac{1-\nu^{\prime 2}}{E^{\prime}},
$$

where $E, E^{\prime}$ and $\nu, \nu^{\prime}$ design, respectively, the Young modulus and the Poisson ratio for the sample and for the tip. From

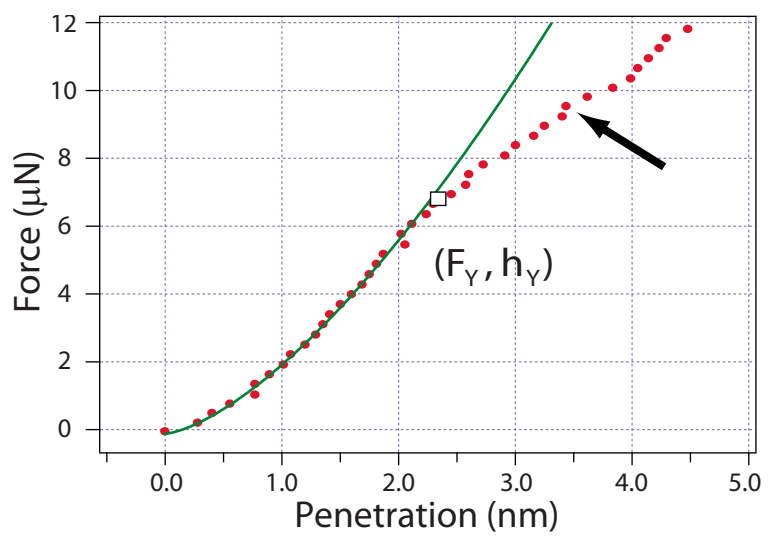

FIG. 4. (Color online) Zoom of the elastic region of Fig. 1 with superimposed (continuous line) Hertzian fit. Arrow marks one of the pop-ins. the experimental curve, we obtain $E^{*}=230 \pm 30 \mathrm{GPa}$, resulting in an intrinsic value for rutile of $E=268 \pm 30 \mathrm{GPa}$. Although the scattering in the measured values is high, we find that our value is consistent with recently reported value of $E^{*}=245 \mathrm{GPa}^{14}$ These values are also consistent with the elastic modulus values of isotropic $\mathrm{TiO}_{2}$, which can be obtained from a Reuss-Voigt average as $287 \mathrm{GPa}$, but is distinctly smaller than the anisotropic values obtained for the (110) face from compression tests in single crystals. ${ }^{15}$ Microscopic images obtained by AFM are also revealing. This first region of the penetration curve is characterized by an absence of permanent traces in the AFM image once the tip has been retracted. This can be seen in the first two indentations in the first row of the $3 \times 3$ matrix of Fig. 1(d). Within the resolution of the technique, no contrast is perceived at the nominal sites of those two first indents. On the basis of the information obtained from both AFM images and Hertzian behavior of the penetration curve, we ascribe this first region of the penetration curve to an elastic stage.

We want to remark that we obtain our values for $E$ directly from the analysis of the elastic stage. This is a different procedure from the one used in most of the published indentation work on oxides, in which the elastic constants are obtained from a measurement of the initial slope of the unloading curve with maximum penetrations well into the plastic stage. The latter follows the method first proposed by Oliver and Pharr ${ }^{16}$ and implies some assumptions about the loading-unloading processes in elastoplastic materials. The good agreement between the values obtained from either approach is encouraging.

\section{Yield point}

The end of the elastic range is characterized by a number of discontinuities in the penetration curve (see Fig. 4). They are similar to discontinuities well reported at the onset of plasticity in metals, ${ }^{2,17}$ which have been baptized pop-ins. Analysis based on AFM images has also shown that these pop-ins are to be associated with the generation of a certain type of dislocation loops. ${ }^{18}$ Note that these observations are not restricted to metals: a similar correspondence between pop-ins and dislocation activity has been described in alkali halides. ${ }^{12}$ In view of these analogies, we ascribe the pop-ins of Fig. 4 to the first yield of the oxide. Note that, contrary to our previous work on $\mathrm{Au}$ surfaces, ${ }^{18}$ the lower resolution attained with the $\mathrm{TiO}_{2}$ sample under ambient conditions prevents us from recognizing individual dislocations around the indentation point. The values at which this first yield is observed have a certain degree of variability among the different nanoindentations. Averaging over a number of samples, we obtain $F_{Y}=6.5 \mu \mathrm{N}, h_{Y}=2.2 \mathrm{~nm}$. The uncertainty in these numerical values may be as high as $30 \%$. Although this is probably due to the difficulties associated with preparation of reproducible smooth oxide surfaces, it can be also be related to the intrinsic stochastic character of the dislocation emission process. ${ }^{19}$

Instead of using the external force $F$, most theoretical analyses of mechanical properties employ the external mean stress $p$, defined as the external force divided by the area of the contact circle between tip and sample (projected area of 


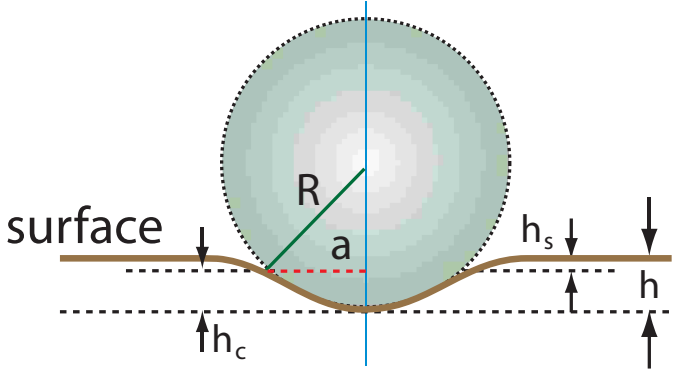

FIG. 5. (Color online) Geometry and basic parameters in spherical indentation in the plastic regime.

contact; see Fig. 5). If the force is normal to the plane surface we may call $p$ the mean pressure. Assuming a continuum, and mediating simple geometrical procedures, from the value of $p$ one can derive a value for the maximum resolved shear stress $^{13}$ of $\tau=0.48 p$. The mean pressure can be written as a function of the directly accessible parameters, $F$ and $h$, in the following way. For not too deep penetrations, $h<R$, the tip can be approximated by a sphere of radius $R$ and one can write $p=F / \pi a_{c}^{2}$, where $a_{c}$ is the radius of the contact circle. Simple geometry, together with the assumption that $R \gg h_{c}$ leads also to $a_{c}^{2} \approx 2 R h_{c}$, where $h_{c}$ is the contact depth. Experimentally, one has no direct access to $h_{c}$ but can relate its value to directly accessible physical quantities as follows. We first write $h_{c}$ in terms of the total penetration depth $h$ and the displacement of the surface at the point of contact, $h_{s}$, as $h_{c}=h-h_{s}$. Now, we use Hertzian theory which relates $h_{s}$ to the directly measurable total penetration depth $h$ through the simple relationship ${ }^{13}$

$$
h_{s}=h / 2 .
$$

One can, then, write

$$
p=\frac{F}{\pi R h} .
$$

By means of Eq. (4) and using the experimental values at the yield point $p_{Y}$ and $h_{Y}$, we can derive the value of the mean pressure $p_{Y}$ at the yield point, $p_{Y}=20 \pm 4 \mathrm{GPa}$. From this $p_{Y}$, we can calculate the value of the maximum resolved shear stress ${ }^{13}$ as $\tau_{Y}=9.8 \pm 2 \mathrm{GPa}$. As the bulk value of the rutile shear modulus is $\mu=112 \mathrm{GPa}$, this implies that $\tau_{Y}$ is about $0.11 \mu$. We want to remark that $\tau_{Y}$ is on the order of $\tau^{\text {theor }}$, the latter being the theoretical value for the critical resolved shear stress in an ideal crystal. Indeed, simple textbooks reasonings estimate $\tau^{\text {theor }}=\mu / 2 \pi$ and more elaborated calculations do not substantially alter its order of magnitude. Note that this similarity between the experimental $\tau_{Y}$ and the theoretical $\tau^{\text {theor }}$, in indentations involving small volumes, resembles the one reported previously in metals nanoindented to a depth of a few nanometers. ${ }^{2,18,20}$ In the latter case, a mechanism involving shear slip of perfect planes has been advanced and it is legitimate to propose that the rutile surface first yield proceeds by a not too distant mechanism.

\section{Beyond the yield point}

Beyond the yield point, we note a first transition regime in the approximate range $2.5<h<11 \mathrm{~nm}$, which ends up in a sharp bend (Fig. 1). In this range, for maximum penetration depths $h$ exceeding $h_{Y}$, we have carried out an AFM study of the potential modifications left over the surface upon tip retraction. Contrary to the observations made in the elastic stage, in the transition regime permanent traces are left on the surface as shown in (Fig. 1). This irreversibility, along with the departure from a Hertzian behavior, implies that this part of the curve already corresponds to an incipient stage of plasticity.

Beyond the transition regime, for penetrations in the range $11<h<40 \mathrm{~nm}$, we find a region (shadowed region in Fig. 3) in which the curve $F$ vs $h$ follows approximately a straight line. Averaging on a number of experiments, we obtain a best value for the slope $\alpha=1.1 \pm 0.1 \mu \mathrm{N} \mathrm{nm}^{-1}$. Imaging the surface after tip retraction by AFM shows that permanent concave traces are left, their size increasing with higher loads [see Fig. 1(d)]. The permanent traces are not, apparently, qualitatively different from those of the transition regime. Note that material dislodged from the sample is clearly visible around the indentation and that its distribution is not isotropic.

We argue now that the straight section of Fig. 3 corresponds to an incipient plastic regime with a constant hardness $H$. Hardness can be defined, in a similar way to the contact pressure, ${ }^{21}$ as $H=F / \pi a_{c}^{2}$. Now, the simple relationship Eq. (3), valid for the elastic stage, does not hold any longer. Instead, one has to make a separate derivation to account for the elastic component of the total deformation in the plastic stage. Following the well-known analysis of Oliver and Pharr, ${ }^{16}$ this component can be written as $h_{s}$ $=\varepsilon F / S$, where the parameter $\varepsilon=0.75$ for paraboloids and $S$ is the stiffness at the peak load. We finally get

$$
H=\frac{F}{2 \pi R h(1-\varepsilon F / h S)},
$$

where $S$ is usually obtained by measuring the initial slope of the unloading curve measured at the maximum penetration.

We have discussed that in the region under scrutiny, our experimental data show that $F / h$ is approximately constant. From the analysis of Ref. 16, the slope $S=2 E^{*}(A / \pi)^{1 / 2}$, where $A$, the section of the contact circle, is $A=2 \pi R h_{c}$. Therefore, as a first approximation, for sufficiently high penetration, the second term in the parentheses in Eq. (5) can be neglected, leading to the relationship,

$$
F \approx 2 \pi R H h .
$$

We associate Eq. (6) to the straight section of the experimental curve of Fig. 3, which can be expressed as $F-F_{o}$ $=\alpha h$. To make them fully consistent, a depth-dependent term can be added to the hardness, $H=H_{o}+(C / h)$, where $C$ is a constant. The term $C / h$ can be physically understood in terms of a moderate indentation size effect (ISE). ${ }^{22}$ Using the above expressions, the extrapolated hardness $H_{o}$ can be esti- 


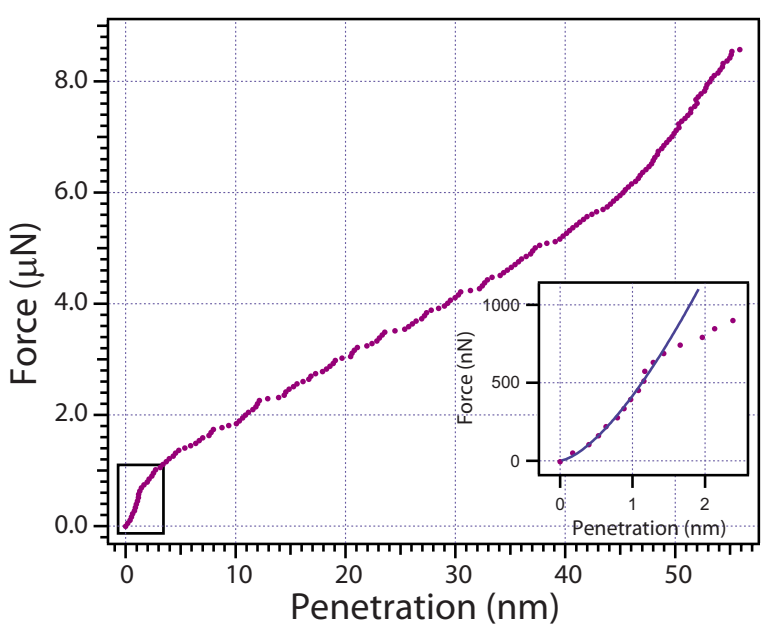

FIG. 6. (Color online) Nanoindentation of $\mathrm{Au}(001)$ with a Si tip of radius $R=23 \mathrm{~nm}$. The Hertzian elastic stage ends up at $h$ $=1.4 \mathrm{~nm}$. The wide linear stage, up to a penetration of $40 \mathrm{~nm}$, corresponds to a constant hardness.

mated from the experimental value of $\alpha$ leading to $H_{o}$ $\approx 3.9 \pm 0.8 \mathrm{GPa}$ where errors in the estimation of the tip radius have been added.

Indentation of oxides has been performed in earlier work $^{14,23-25}$ in order to probe their mechanical properties. Most of these studies could be regarded as microindentations-although, at times, they self-nominate themselves as nanoindentations - their penetrations extending well into the micrometer range. Often a Berkovich-type indenter, with pyramidal or related shape, has been used. In this kind of indentations, the elastic stage is practically not resolved, and geometry is different from that of a spherical tip, leading to a linear relationship between $a_{c}$ and $h$. As a result, the hardness is expected to depend quadratically on $h$. Many authors ${ }^{8,23,25}$ indeed report on this dependence. Quantitatively, the relationship $F=\xi H h^{2}$ holds, where $\xi$ is a dimensionless numerical parameter; for the geometry of a Berkovich indenter $\xi=25.4$. Measuring the curvature of the experimental curve $F$ vs $h$ leads directly to an estimation of $H$. Note that, when compared to our data reported here, these works involve much deeper penetrations, which are likely to probe the bulk of the sample. The values obtained for this hardness well into the bulk, which we shall call $H_{B}$, are around $13 \mathrm{GPa},{ }^{14}$ about a factor of 3 larger than the value that we have obtained for $H_{o}$ in nanoindentations involving small penetration volumes.

\section{B. Gold}

We report now penetration curves for $\mathrm{Au}(001)$, obtained under conditions quite similar to the ones prevailing for $\mathrm{TiO}_{2}$. Worth emphasizing are the similar shapes of the penetration curves in both materials. The initial elastic stage of nanoindented $\mathrm{Au}$ is sufficiently documented and will not be discussed again here. ${ }^{2,18}$ We just remind that a Hertzian behavior is always apparent and that pop-ins mark the end of the elastic stage. From the data of Fig. 6, using Eq. (4) the corresponding mean pressure at the yield point can be esti- mated as $p_{Y} \approx 5.4 \pm 0.5 \mathrm{GPa}$. Errors are here smaller than in the case of $\mathrm{TiO}_{2}$. This value of $p_{Y}$ is in good agreement with the data of Kiely and Houston. ${ }^{2}$ Let us now concentrate at the onset of the elastic stage. As shown in the figure, the transit from elastic Hertzian behavior to plasticity is identified by a sharp bend in the penetration curve. After the yield point, a wide region in the shape of a straight line is apparent. For penetrations above $h \geqslant 40 \mathrm{~nm}$, the curve bends up. This is interpreted as the end of the region of shallow depth when the penetration $h$ begins to be comparable to the radius $R$ and the tip can no longer be approximated to a sphere. Note that, for higher penetrations, it is expected that $F \propto h^{2}$. From the slope of the straight portion of the curve, using Eq. (6), we obtain a hardness $H_{o}=0.8 \pm 0.2 \mathrm{GPa}$. Reports of the hardness of pure gold in the bulk are not frequent but we can use a handbook value of $0.25<H_{B}<0.35 \mathrm{GPa}$. This compares well with values in another noble metal, of $H_{B}$ $=0.34 \mathrm{GPa}$ in silver. ${ }^{26}$ Contrary to the case of the oxide, the hardness which we have measured in gold from nanoindentations exceeds the hardness of bulk Au by more than a factor of 2 .

\section{HARDNESS OF OXIDES}

In this section, we argue that the mechanical behavior of nanoindentations in both $\mathrm{Au}$ and $\mathrm{TiO}_{2}$ can be rationalized as follows. We define an effective volume, which can be imagined as the volume affected by the indentation, i.e., the volume in which the stress induced by the external load exceeds a certain threshold. In fact, microindentations and nanoindentations can be differentiated by reference to their effective volumes. When the effective volume is small-in the following, we restrict the name nanoindentation to this limit—no pre-existing dislocations are encompassed and the material responds as an ideal crystal. On the contrary, when the effective volume is large-in the following, this is related to microindentations - the crystal has a mechanical response corresponding to that of the bulk.

Having this concept in mind, we refer now to three types of hardness (we are actually extending somehow the concept of hardness to design the mean contact pressure of the external load), which we discuss in the present paper: the first two types correspond to mechanical response associated to small effective volume whereas the third one corresponds to bulk response. The first type of hardness is the yield strength $p_{Y}$, associated to defect nucleation at the surface in the pristine material. The second type of hardness is $H_{o}$, which we name in the present paper surface hardness, is observed when preexisting defects are already available in the material but still very small volumes are affected by the applied stress due to the nanoindenter. This condition is achieved just if the nanoindenter radius is on the order of a few tenths of nanometers. Finally, the third type is the well-known bulk hardness $H_{B}$, usually obtained with microindentations and taken as a reference. It is associated to pre-existing defects set into motion or multiplication.

Now, we discuss, in this light, the yield strength and hardness of $\mathrm{TiO}_{2}(110)$ and its comparison with Au. First, we compare for both materials the values of their yield strength 
$p_{Y}$; second, we analyze the hardness $H_{o}$ at the onset of plasticity of the nanoindented surface in $\mathrm{TiO}_{2}$ and we discuss its similarities to that of $\mathrm{Au}$; and, third, we discuss why $H_{o}$ is much smaller than $H_{B}$ in $\mathrm{TiO}_{2}$, whereas the opposite is true of $\mathrm{Au}$.

\section{A. Yield strength $p_{Y}$}

The measured values of the yield strength $p_{Y}$ appear to be strongly dependent on the size of the volume which is actually explored by the indentation. As is well known, ${ }^{27}$ when the bulk is probed in metals-in the case of indented surfaces, it would be the realm of microindentations-the experimental value for the critical shear stress $\tau_{Y}^{\text {bulk }}$ becomes orders of magnitude smaller than $\tau^{\text {theor }}$. This is usually explained in terms of pre-existing dislocations. Yield proceeds via movements of these dislocations, which have an energetic cost much lower that that of nucleating new dislocations. On the other hand, our results above indicate that when only a small volume around the indentation point is explored-nanoindentations-the response is quite different. The yield strength of gold becomes on the order of the theoretical value for slipping of a plane on its adjacent one in an ideal crystal $\left(\tau^{\text {theor }}\right) .^{2,18}$ We can account for this hardening by arguing that when the effective volume is smallnanoindentation-no pre-existing dislocations are present therein. As a consequence, yield takes place when the maximum resolved shear stress is comparable to $\tau^{\text {theor }}$. As the effective volume increases-microindentation-it encompasses more and more pre-existing dislocations and the metal begins to yield at a shear stress substantially lower, $\tau_{Y}^{\text {bulk }} \ll \tau_{Y}^{\text {theor }}$, because yield does no longer require nucleation of new dislocations, but making the pre-existing ones glide. The latter mechanism involves much lower stresses (basically, the Peierls stress).

Turning now to oxides: sufficiently large effective volumes do not result in softening of the sample - as in the case of metals-because pre-existing dislocations are likely to be pinned. Yield would require, then, nucleation of new dislocations, much in the same way as in the domain of small effective volumes where crystal conditions can be described as those of an ideal crystal. On the basis of our model, we would not expect, then, much difference between the yield point at microindentations and nanoindentations, as indeed is observed. For indentations involving very large tip radii (tens or hundreds of micrometers), ${ }^{5}$ the effective volume is likely to encompass pre-existing microcracks and one ends up with the well-known brittle response in the bulk.

\section{B. Surface hardness at the onset of plasticity, $H_{o}$}

To gain insight into the mechanisms operating during the incipient stages of plasticity in surface nanoindentation, we compare our data of yield stress $p_{Y}$ and surface hardness $H_{O}$ with the corresponding ones in gold. The set of data for both materials involves the same small effective volumes and is obtained with the same experimental apparatus. Two similarities are patent (compare Figs. 3 and 6): (1) the $F$ vs $h$ penetration curves have the same shape in the two cases: a Hertz-type region followed by a sharp bend and a linear

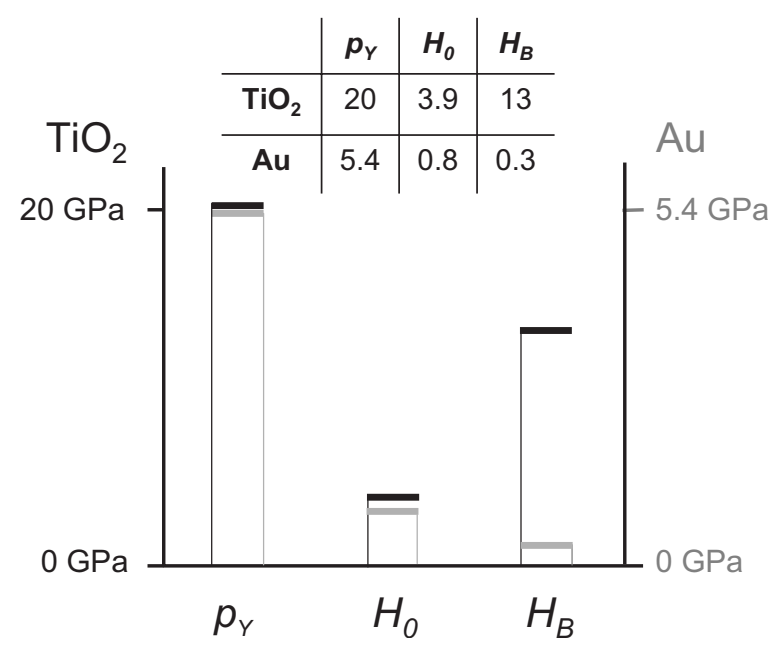

FIG. 7. Comparison of the yield strength $p_{Y}$, surface hardness $H_{o}$, and bulk hardness $H_{B}$ values in $\mathrm{TiO}_{2}$ (left axis, black) and $\mathrm{Au}$ (right axis, gray). We have normalized their corresponding scales of $p_{Y}$ to better compare them.

stage corresponding to a $H_{o}=0.8 \mathrm{GPa}$ in $\mathrm{Au}$ and $H_{o}=3.9 \mathrm{GPa}$ in $\mathrm{TiO}_{2}$. (2) If we define a parameter $r$ measuring the discontinuity in $p$ at the yield point, $r=p_{Y} / H_{o}$, we have rather similar values for both types of material, $r=5.3$ for $\mathrm{TiO}_{2}$ and $r=6.8$ for Au. These affinities call for a common origin of both processes.

In the bulk, hardness in the plastic stage, measured by microindentations involving large effective volumes, is commonly interpreted in terms of a Nix and Gao (NG) mechanism. $^{22}$ This mechanism, based on the pioneering ideas of Taylor, ${ }^{27}$ explains the value of hardness in terms of the work hardening of the material due to a mixture of preexisting and geometrically necessary dislocations (GNDs). Often, this leads to the so-called indentation size effect, by which hardness increases at low penetration depths. Evidence of this ISE has been found both in oxides ${ }^{8,25,28}$ and metals. $^{22,29}$ In the case of metals, even probing deep in the bulk (no ISE), the values of $H_{B}$ are larger than the macroscopic yield strength $\tau_{Y}^{\text {bulk }}$ as hardness in the plastic region is controlled by work hardening. In the case of oxides, values of $\tau_{Y}^{\text {bulk }}$ are not easily found as many microindentation studies do not intend to resolve either the elastic region or the yield point, and directly probe the plastic region. Nevertheless, some of the existing values ${ }^{30}$ show that $H_{B}$ are on the order of $\tau_{Y}^{\text {bulk }}$. Note that the values we measured for the yield strength $p_{Y}$ are much larger than $\tau_{Y}^{\text {bulk }}$ and on the order of the theoretical value $\tau^{\text {theor }}$, since we are exploring volumes free of pre-existing dislocations. To assist in the discussion, Fig. 7 shows a scheme of the different levels of hardness referred to in the paper.

The NG mechanism is unlikely to explain the behavior of nanoindentations involving small effective volumes. One might be tempted to explain the region of constant hardness at the onset of plasticity in terms of the Swadener ${ }^{31}$ extension of the NG mechanism to spherical tips. The latter predicts indeed a constant hardness, but its value would be far too high and difficult to reconcile with our measured values. Much more difficult to explain would be the fact that, con- 
trary to the case of microindentations, the hardness one measures at low effective volumes is now smaller than the yield strength $H_{B}<p_{Y}$. In fact, there is a sudden decrease in hardness at the yield point in $\mathrm{TiO}_{2}$ - as quantified by a value of $r=5.3$ - which differs strikingly from the $r \approx 1.5$ of the bulk $\mathrm{TiO}_{2}$ and of values even of $r<1$ in other oxides. ${ }^{30}$ In the case of metals too, whereas $r=6.8$ for small effective volumes, its value is about $r=1 / 3$ in the bulk.

A confirmation of our views derives from recent work by Scholz et al. ${ }^{28}$ in nanoindented $\mathrm{BaTiO}_{3}$. In agreement with our results in $\mathrm{TiO}_{2}$, these authors reported a clear discontinuity at the yield point, corresponding to a high value of $r$ $\approx 2.5$. They also reported a different response of oxide surfaces to indentations involving different volumes. When the volumes involved in their experiments are larger than ours by a factor in the range of $10^{2}-10^{5}$, they can explain their results in terms of an ISE resulting from work hardening as described by the NG model. However, they recognized that their explanation in terms of an ISE seems to break down when their indentation tip radii become small (less than double than ours). Their results are in good agreement with ours if one assumes that when they use this small radius, they are reaching the region where the effective volume is small enough to make a switch into the ideal crystal conditions. Under the latter conditions, there is no ISE because the hardness is dominated by a different physical process, i.e., dislocation emission.

We need, therefore, a different mechanism to account for the reduced hardness of both $\mathrm{Au}$ and $\mathrm{TiO}_{2}$ in the early stages of plasticity when effective volumes are small enough. As there is ample evidence of the operation of dislocation sources (Frank-Read and other types) with low-energy barriers in metals, we believe that our results point to a comparable origin for the low hardness observed at the initial stages of plasticity in the oxide. We propose, then, the following picture for the onset of plasticity in the oxide surface: as the external load increases, so does the average pressure. Once the resolved shear stress attains a certain threshold value - on the order of $\tau_{Y}$ - the crystal yields and dislocation loops are generated. Then, the pressure is partially released and a low-energy-barrier dislocation loop source begins to operate. Comparing $\mathrm{Au}$ and $\mathrm{TiO}_{2}$, we remark that in the nanoindentation region, the ratio between the respective hardnesses $H^{\mathrm{ox}} / H^{\mathrm{Au}} \approx 5$. Although other parameters may be more relevant, note that the stress necessary to operate a dislocation source scales with $\mu$, and that $\mu^{\mathrm{ox}} / \mu^{\mathrm{Au}} \approx 4.2$.

A mechanism not afar from the one proposed above has been suggested in earlier work on $\mathrm{MgO}$ (Ref. 23) to account for the shape of the penetration curve. It is worth pointing out that our proposal implies that the physical process hardening the material is now dislocation emission. The deformation process is controlled by the emission of dislocations, proceeding after the nucleation of the first loops at the yield point, through the operation of a low-barrier loop source. The fact that $H_{o}$ is definitely smaller than $p_{Y}$ strongly suggests the operation of a dislocation source ignited by a much smaller stress than the one corresponding to the first yield. We want to remark that this mechanism operates at small effective volumes and does not contradict the operation of the NG mechanism in the usual range of large effective volumes.

\section{Reduced surface hardness $\mathrm{H}_{o}$ in $\mathrm{TiO}_{2}$}

We turn now to the different behaviors of $\mathrm{Au}$ and $\mathrm{TiO}_{2}$ regarding the ratio $q$ between their hardnesses in the surface region and in the bulk, $q=H_{o} / H_{B}$. Whereas in Au the nanoindented surface region is harder than the bulk, $q_{\mathrm{Au}} \approx 2$, the opposite is true in $\mathrm{TiO}_{2}, q_{\mathrm{ox}} \approx 1 / 3$. We write approximative values because there is a large scattering in the measurements in the bulk. We offer the following explanation: in the nanoindentation process, for both metal and oxide surfaces, the effective volume that we probe is small enough to avoid encompassing any pre-existing dislocations. Under these conditions, following the nucleation of the first loop (yield point), the hardness in the region of constant low hardness corresponds to the operation of a low-barrier dislocation source. In the case of $\mathrm{Au}$, as the tip excursion progresses into the bulk, pre-existing dislocations able to glide start being activated. The hardness is now controlled, Taylor-type, by the work hardening related to the dislocation density. As is well known, this results a rather low value of $H_{B}$, smaller than the one corresponding to the operation of the loop source, and one ends up with a value of $q \gg 1$. On the other hand, $\mathrm{TiO}_{2}$ is 2 orders of magnitude harder than $\mathrm{Au}$ in the bulk. This is probably due to the fact that in the bulk of $\mathrm{TiO}_{2}$, pre-existing dislocations are pinned and stresses cannot make them glide. The reason why low-barrier dislocation sources, similar to the ones proposed to control hardness in the low effective volume limit, do not seem to operate in the bulk is not yet clear. One can advance the following explanation: dislocations introduced by the source can interact with preexisting (pinned) dislocations - or even among themselvesresulting in very immobile locks, which further impede the motion of subsequently nucleated dislocations. If the effective volume is small, no pre-existing dislocations exist in it and the locking does not take place.

\section{CONCLUSIONS}

Three are the main conclusions of the present work. The most important one is that, for both $\mathrm{Au}$ and $\mathrm{TiO}_{2}$, there is an incipient plastic region of deformation of the surface which can be described in terms of a constant hardness $H_{o}$ different from the hardness measured in the bulk, $H_{B}$. This strikingly similar behavior of both materials can be explained in terms of a model in which the nucleation of new dislocation loops at the yield point is followed by the operation of a dislocation source with a low activation barrier. Operation of this barrier requires a much lower flow stress than the one corresponding to yield. In the case of $\mathrm{TiO}_{2}$, we have observed an enhanced plasticity for low tip penetrations, which suggests that dislocation activity in this material largely depends on whether applied stress is localized in nanometric volumes. A second conclusion is that by introducing the concept of effective volume, a unifying interpretation can be given of the response to indentation under two different conditions: nanoindentations, involving short penetrations which affect rather small volumes of the sample, and microindentations, which explore large volumes representative of the bulk of the sample. Using this concept, together with the tendency for the dislocation to get pinned in $\mathrm{TiO}_{2}(110)$, we explain why 
in $\mathrm{TiO}_{2}(110)$ the surface hardness $H_{o}$ is about one-third of the bulk hardness $H_{B}$, whereas in gold it is about twice larger. Third, in $\mathrm{TiO}_{2}$, we have characterized a first reversible region, recognized as elastic, in which there are no permanent traces left on the surface after tip retraction. Fitting a Hertzian shape to the curve allows a determination of the Young modulus which results in values of the latter comparable to those determined more conventionally from the initial slope of unloading curves in the plastic stage. In addition, we have also shown the validity of the AFM as a valid technique for quantifying elastic properties and yield strength in oxides directly form the loading curve. The conclusions drawn here regarding the reduced plasticity at small explored volumes in $\mathrm{TiO}_{2}$ may have deep implications when dealing with plastic deformation in the surface regions or with nanophase materials, where stresses can be concentrated in very small volumes due to surface, interface, or nanostructural effects.

\section{ACKNOWLEDGMENTS}

The authors acknowledge financial support from the Comunidad de Madrid Project No. CAM-S-0505/PPQ/0316, from Santander-UCM Project No.PR27/05-13926, and from the Spanish Ministerio de Educación y Ciencia Project No. MAT2006-13149-C02-01.
*Corresponding author violeta.navarro@ fis.ucm.es

${ }^{1}$ A. C. Fischer-Cripps, Nanoindentation (Springer, New York, 2004).

${ }^{2}$ J. D. Kiely and J. E. Houston, Phys. Rev. B 57, 12588 (1998).

${ }^{3}$ K. J. Hemker and W. N. Sharpe, Jr., Annu. Rev. Mater. Res. 37, 93 (2007).

${ }^{4}$ B. R. Lawn, J. Am. Ceram. Soc. 81, 1977 (1998).

${ }^{5}$ Y.-W. Rhee et al., J. Am. Ceram. Soc. 84, 561 (2001).

${ }^{6} \mathrm{P}$. Gumbsch, S. Taeri-Baghbadrani, D. Brunner, W. Sigle, and M. Ruhle, Phys. Rev. Lett. 87, 085505 (2001).

${ }^{7}$ B. R. Lawn et al., Science 263, 1114 (1994).

${ }^{8}$ Z. Peng, J. Gong, and H. Miao, J. Eur. Ceram. Soc. 24, 2193 (2004).

${ }^{9}$ R. Nakamura et al., J. Am. Chem. Soc. 127, 12975 (2005)

${ }^{10}$ I. Horcas et al., Rev. Sci. Instrum. 78, 013705 (2007).

${ }^{11}$ R. J. Cannara, M. J. Bruckman, and R. W. Carpick, Rev. Sci. Instrum. 76, 053706 (2005).

${ }^{12}$ T. Filleter, S. Maier, and R. Bennewitz, Phys. Rev. B 73, 155433 (2006).

${ }^{13}$ K. L. Johnson, Contact Mechanics (Cambridge University Press, Cambridge, 1985).

${ }^{14}$ K. Kurosaki et al., J. Alloys Compd. 386, 261 (2005).

${ }^{15}$ H. Li and R. C. Bradt, J. Mater. Sci. 28, 917 (1993).
${ }^{16}$ W. C. Oliver and G. M. Pharr, J. Mater. Res. 7, 1564 (1992).

${ }^{17}$ J. Li et al., Nature (London) 418, 307 (2002).

${ }^{18}$ A. Asenjo, M. Jaafar, E. Carrasco, and J. M. Rojo, Phys. Rev. B 73, 075431 (2006).

${ }^{19}$ C. A. Schuh, J. K. Mason, and A. C. Lund, Nature Mater. 4, 617 (2005).

${ }^{20}$ V. Navarro, O. Rodríguez de la Fuente, A. Mascaraque, and J. M. Rojo, Phys. Rev. Lett. 100, 105504 (2008).

${ }^{21}$ Sometimes, the concept of hardness is extended to denote the mean pressure in the elastic region; the yield strength is, then, the hardness at the yield point.

${ }^{22}$ W. D. Nix and H. Gao, J. Mech. Phys. Solids 46, 411 (1998).

${ }^{23}$ J. Woirgard et al., J. Eur. Ceram. Soc. 18, 2297 (1998).

${ }^{24}$ M. J. Mayo et al., J. Mater. Res. 5, 1073 (1990).

${ }^{25}$ G. Feng and W. D. Nix, Scr. Mater. 51, 599 (2004).

${ }^{26}$ Q. Ma and D. R. Clarke, J. Mater. Res. 10, 853 (1995).

${ }^{27}$ A. H. Cottrell, Dislocations and Plastic Flow in Crystals (Clarendon, Oxford, 1953).

${ }^{28}$ T. Scholz et al., Appl. Phys. Lett. 88, 091908 (2006).

${ }^{29}$ K. Durst et al., Acta Mater. 54, 2547 (2006).

${ }^{30}$ M. Fujikane et al., J. Alloys Compd. 431, 250 (2007).

${ }^{31}$ J. G. Swadener, E. P. George, and G. M. Pharr, J. Mech. Phys. Solids 50, 681 (2002). 